\title{
Dysopsonin Activity of Serum DNA-Binding Proteins Favorable for Gene Delivery
}

\author{
Feng Liu, Amber Frick, Xing Yuan, and Leaf Huang \\ Division of Molecular Pharmaceutics, Eshelman School of Pharmacy, University of North Carolina, Chapel Hill, North Carolina \\ (F.L., A.F., L.H.); and Duke University School of Medicine, Durham, North Carolina (X.Y.)
}

Received July 31, 2009; accepted October 27, 2009

\begin{abstract}
Naked DNA is regarded as the safest and simplest method of gene delivery. However, normally intravenously injected naked plasmid DNA is rapidly eliminated from the blood. It has been hypothesized that opsonins, a category of serum DNA-binding proteins (SDBPs), label the injected plasmid DNA as foreign so that it may be recognized and rapidly removed from the bloodstream by liver nonparenchymal cells. Contrary to the hypothesis, our data indicate that some SDBPs across multiple species may have important dysopsonin properties, acting to reduce liver uptake. Formation of SDBP and DNA complexes was observed by agarose gel electrophoresis. An in vivo study
\end{abstract}

involving hepatic artery and portal vein occlusion in a mouse model confirmed the activity of serum diminishing liver uptake of DNA. Data using hydrodynamic gene transfer in the mouse liver and in situ transfection in the mouse lung revealed that serum proteins bound to DNA do not affect the biological activity of the plasmid DNA. We have identified several SDBPs with potential dysopsonin properties. The SDBPs with dysopsonin properties and DNA complexes may be further modified and ultimately be developed into a novel DNA carrier system favorable for systemic gene delivery.
Although nonviral vectors have been designed for systemic gene therapy with increased stability in the bloodstream, problems such as aggregation and inflammatory toxicity exist. With the administration of these vectors, inflammation is characterized by increases in inflammation-inducing cytokines, including tumor necrosis factor $\alpha$, interleukin 12, interleukin 6, and interferon $\gamma$ (Sellins et al., 2005). Injection of naked plasmid DNA is regarded as the safest and simplest method of gene delivery. However, normally, intravenously injected naked plasmid DNA is rapidly eliminated from the blood and largely taken into liver nonparenchymal cells (Kawabata et al., 1995; Yoshida et al., 1996; Nakamura et al., 1998; Hisazumi et al., 2004), primarily endothelial cells and not Kupffer cells where it is degraded (Liu et al., 2007), a process that, in general, results in no gene expression. This endothelial barrier to transfusion may be surmounted by manual liver massage (Liu and Huang, 2002) similar to the hydrodynamic-based transfection method studied in our laboratory and others and enlargement of the fenestrae allowing transfection of hepatocytes (Budker et al., 1998; Liu et al.,

This work was supported by the National Institutes of Health [Grants DK065964, CA129835].

Article, publication date, and citation information can be found at http://jpet.aspetjournals.org.

doi:10.1124/jpet.109.159541.
1999; Zhang et al., 1999; Kobayashi et al., 2001; Lecocq et al., 2003; Zhang et al., 2004). In an in vivo mouse model, the liver could take up over $50 \%$ of $5 \mu \mathrm{g}$ of perfused plasmid DNA with a maximum $25 \mu \mathrm{g}$ of plasmid DNA during a single pass with a slower clearance rate compared with the rate of uptake. Once the liver becomes saturated with plasmid DNA, additional naked DNA is available to transfect other organs. Understanding the kinetics of DNA clearance after intravenous injection is imperative to defining dosing parameters and the sustained utilization of naked DNA as a gene therapy vector (Liu et al., 2007). It is well known that some serum DNA-binding proteins (SDBPs) or nucleases also degrade plasmid DNA or are responsible for DNA clearance via the intravenous route (Kawabata et al., 1995). We proposed here that SDBPs may be involved in the liver uptake process as opsonins.

Opsonins, a category of some serum proteins that enhance phagocytosis by the reticuloendothelial system, have been suggested to label the injected plasmid DNA as foreign so that it may be recognized, removed from the bloodstream, and degraded by nonparenchymal cells. In addition, opsonins have been implicated in liver uptake of liposomes (Moghimi and Patel, 1989). Serum proteins previously identified as having opsonin effects include IgG, albumin, complement C3, and various apolipoproteins (Yan et al., 2005). 
We originally hypothesized that SDBPs would bind to injected plasmid DNA, labeling plasmid DNA as foreign and resulting in uptake by liver nonparenchymal cells. Contrary to the original hypothesis, additional data indicate that some SDBPs may have important dysopsonin properties, acting to reduce liver uptake, and protective properties against blood degradation of plasmid DNA. We purified and identified potential proteins with dysopsonin properties for future gene delivery investigation. The relatively simple, nonviral gene delivery system of natural serum proteins coupled with DNA may aid with extending serum circulation time, reducing toxicities inherent with lipoplex delivery, and improving delivery efficiency.

\section{Materials and Methods}

Liver Single-Pass Perfusion System. The single-pass perfusion system developed by us (Liu et al., 1995) was used to test plasmid DNA uptake by the liver. In brief, plasmid DNA (with or without preincubation of serum) containing $1 \%{ }^{125}$ I-labeled plasmid DNA was added with $2 \mathrm{ml}\left(\mathrm{pH} 7.4,37^{\circ} \mathrm{C}\right)$ of Krebs-Henseleit buffer, and then perfused via the portal vein through the mouse liver (five mice per group), which was previously prewashed with $3 \mathrm{ml}$ of buffer (prewarmed to $\left.37^{\circ} \mathrm{C}\right)$. Mice (18-20 g body weight) were anesthetized by 2,2,2-tribromoethanol $(0.6 \mathrm{ml}, 20 \mathrm{mg} / \mathrm{ml}$ i.p. $)$ during the experiment. The inferior vena cava was cut at the beginning of the experiments to drain the blood and immediately followed by washing. Unbound plasmid DNA in the liver was removed by washing the perfused liver with $5 \mathrm{ml}$ of buffer via the same route. The perfusion rate was kept constant at $2 \mathrm{ml} / \mathrm{min}$. The amount of plasmid DNA taken up by the liver was determined by measuring the ${ }^{125} \mathrm{I}$ radioactivity in the liver. The serum was prepared just before use as follows: mouse blood was collected by retro-orbital bleeding and allowed to clot at room temperature for $20 \mathrm{~min}$, then it was centrifuged at $2000 \mathrm{~g}$ for $20 \mathrm{~min}$ at $4^{\circ} \mathrm{C}$, and the serum (supernatant) was collected.

Iodination and in Vivo Distribution of DNA. Plasmid DNA was labeled with ${ }^{125}$ I according to a published method (Zhang et al., 2006). In brief, in an IODO-GEN precoated iodination tube (Pierce Chemical, Rockford, IL), the following reagents were added: $50 \mu \mathrm{l}$ of $2 \mu \mathrm{g} / \mu \mathrm{l} \mathrm{DNA}, 30 \mu \mathrm{l}$ of $0.35 \mathrm{M}$ sodium acetate, $\mathrm{pH} 4.0$, and $600 \mathrm{pmol}$ of ${ }^{125} \mathrm{I}$-labeled sodium iodine. The mixture was incubated at $50^{\circ} \mathrm{C}$ for $30 \mathrm{~min}$. After chilling on ice, $10 \mu \mathrm{l}$ of $1 \mathrm{~N} \mathrm{NaOH}$ was added, and then free iodine was removed by use of a Bio-Spin 30 column (Bio-Rad Laboratories, Hercules, CA). After injection of DNA $\left({ }^{125} \mathrm{I}\right.$-labeled DNA diluted 1:100 with unlabeled plasmid DNA), the mice were killed at $2 \mathrm{~min}$, and the organs, such as the lung, liver, spleen, and blood, were collected. The collected blood and organs were subjected to assay for ${ }^{125}$ I radioactivity with use of a $\gamma$-counter. The distribution of DNA in the blood was estimated with the assumption that the total amount of blood is approximately $7.3 \%$ of the body weight.

Examination of Dysopsonin Activity in Living Animal Model. Mice of $15 \mathrm{~g}$ body weight were anesthetized with an intraperitoneal injection of $8 \mathrm{mg}$ of 2,2,2-tribromoethanol. Liver was exposed through ventral midline incision. Mice were intravenously (tail vein) injected with plasmid DNA in $100 \mu \mathrm{l}$ saline $(0.9 \% \mathrm{NaCl})$ immediately after occlusion of blood flow through the liver. The injection speed for most experiments was $100 \mu \mathrm{l} / \mathrm{s}$. The occlusion of blood flow was performed by a simple clip at the combination of the portal vein and hepatic artery. The clip was released immediately after injection, and the blood sample and other organs were collected 2 min after the release of the clip.

Purification and Identification of SDBPs. Purification of SDBPS was initiated by use of a dsDNA-cellulose column (GE Healthcare, Little Chalfont, Buckinghamshire, UK) according to manufacturer's protocol. However, a substantial amount of SDBPs remained bound to the DNA-cellulose column after elution of SDBPs with 1 to $2 \mathrm{M}$ $\mathrm{NaCl}$ as detected with the Coomassie Plus Protein Assay Reagent Kit (Pierce Chemical). We developed a new method for identifying SDBPs by digesting DNA (in the dsDNA-cellulose column incubated with mouse serum) with DNase I to release SDBPs. In brief, $200 \mu \mathrm{l}$ of mouse serum was added to $200 \mu l$ of dsDNA-cellulose containing $125 \mu \mathrm{g}$ of DNA and gently vortexed for $2 \mathrm{~min}$. The mixture was centrifuged at $3000 \mathrm{~g}$ to remove the supernatant. Pellets were washed six times with equilibration buffer (10 mM Tris-HCl, $\mathrm{pH} 7.5$, $0.1 \mathrm{mM}$ dithiothreitol, $5 \%$ glycerol (v/v), $0.1 \mathrm{M} \mathrm{NaCl}$, and $0.1 \mathrm{mM}$ EDTA) and then washed three times with phosphate-buffered saline $1 \times$ buffer. All processes above were completed at $4^{\circ} \mathrm{C}$. Subsequently, pellets were incubated with DNase I at $37^{\circ} \mathrm{C}$ for $1 \mathrm{~h}$. To minimize the DNase appearance in the SDS-PAGE gel because the DNase I band may overlap with SDBPs, a low concentration of DNase I $(0.2 \mu \mathrm{g})$ and longer digestion time $(1 \mathrm{~h})$ were used. After digestion, the supernatant was loaded onto a Tris-HCl $12 \%$ polyacrylamide gel (Bio-Rad Laboratories). Proteins were visualized with Coomassie blue staining, and those bands unique to the dsDNA-cellulose and mouse serum lane were identified by mass spectrophotometry at the Hooker Proteomics Center at the University of North Carolina.

\section{Results}

The Role of Serum in Liver Uptake of DNA. In general, intravenous injection of naked plasmid DNA results in no gene expression even in the liver where the highest uptake is observed. Injected plasmid DNA is taken up extensively by liver nonparenchymal cells. After intravenous injection in a mouse model, as shown in Fig. 1, both intact supercoiled plasmid DNA and DNA fragments that were amplified by PCR (Hofman et al., 2001) were distributed mostly to the liver with a small amount remaining in the blood and other compartments, including lung, heart, kidney, and spleen. The liver took up over $50 \%$ of DNA 2 min after injection of $5 \mu \mathrm{g}$ of DNA. Less than $20 \%$ of the injected DNA was detected in blood, and less than $2 \%$ was detected in other organs. A similar uptake pattern existed for both intact plasmid and PCR fragment DNA, which indicates that liver uptake of DNA is independent of the size of DNA. To observe the role of the extent of naked DNA uptake by the liver, a single-pass liver perfusion system was used in a mouse model. It is noteworthy that DNA uptake by the liver was

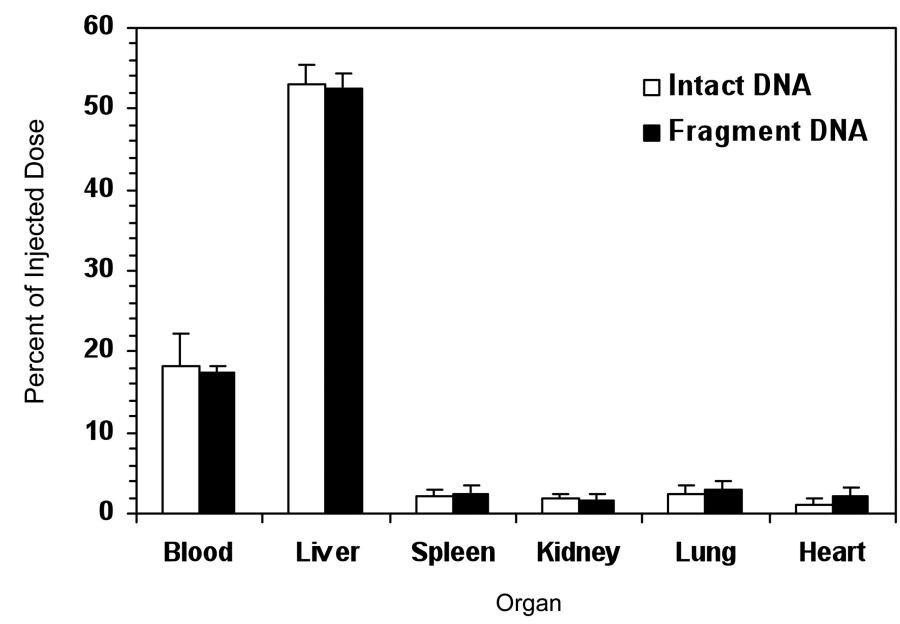

Fig. 1. Distribution of DNA. After intravenous injection in a mouse model, intact supercoiled DNA and DNA fragments were distributed mostly to the liver with a smaller amount of DNA retained in the blood compartment. Minimal amounts of DNA were detected in the spleen, kidney, lung, and heart. 
reduced after perfusion with plasmid DNA incubated with mouse serum compared with perfusion with naked plasmid DNA. Furthermore, increasing the ratio of serum to plasmid DNA during the incubation process resulted in significantly decreased uptake of plasmid DNA when perfused to the mouse liver. When $5 \mu \mathrm{g}$ of plasmid DNA was complexed with $2 \mu \mathrm{l}$ of serum, plasmid DNA uptake was decreased from $60 \%$ (plasmid DNA alone) to $43 \%(p<0.05)$, and further decreased to less than $30 \%(p<0.01)$ at the ratio of $1: 1$ of DNA/serum (Fig. 2a). This decreased liver uptake is not limited to the mouse model, because serum from rat, bovine, and human species also inhibits DNA liver uptake (Fig. 2b). We hypothesized that the decreased uptake of DNA incubated with serum is due to a dysopsonin mechanism in that some serum proteins bind to DNA such that the DNA is protected from being recognized by nonparenchymal cells. Indeed, a gel shift assay demonstrated the formation of SDBP and DNA complexes. These SDBP and plasmid DNA complexes (SDBPs/DNA) formed a distinct band characterized by decreased gel mobility compared with plasmid DNA alone (Fig. 3).

Examination of the Dysopsonin Activity of SDBPs in Vivo. SDBPs theoretically must bind to DNA to exhibit their dysopsonin activity. However, when DNA is directly injected into the bloodstream, SDBPs do not have enough time to bind the DNA because the DNA will be quickly taken
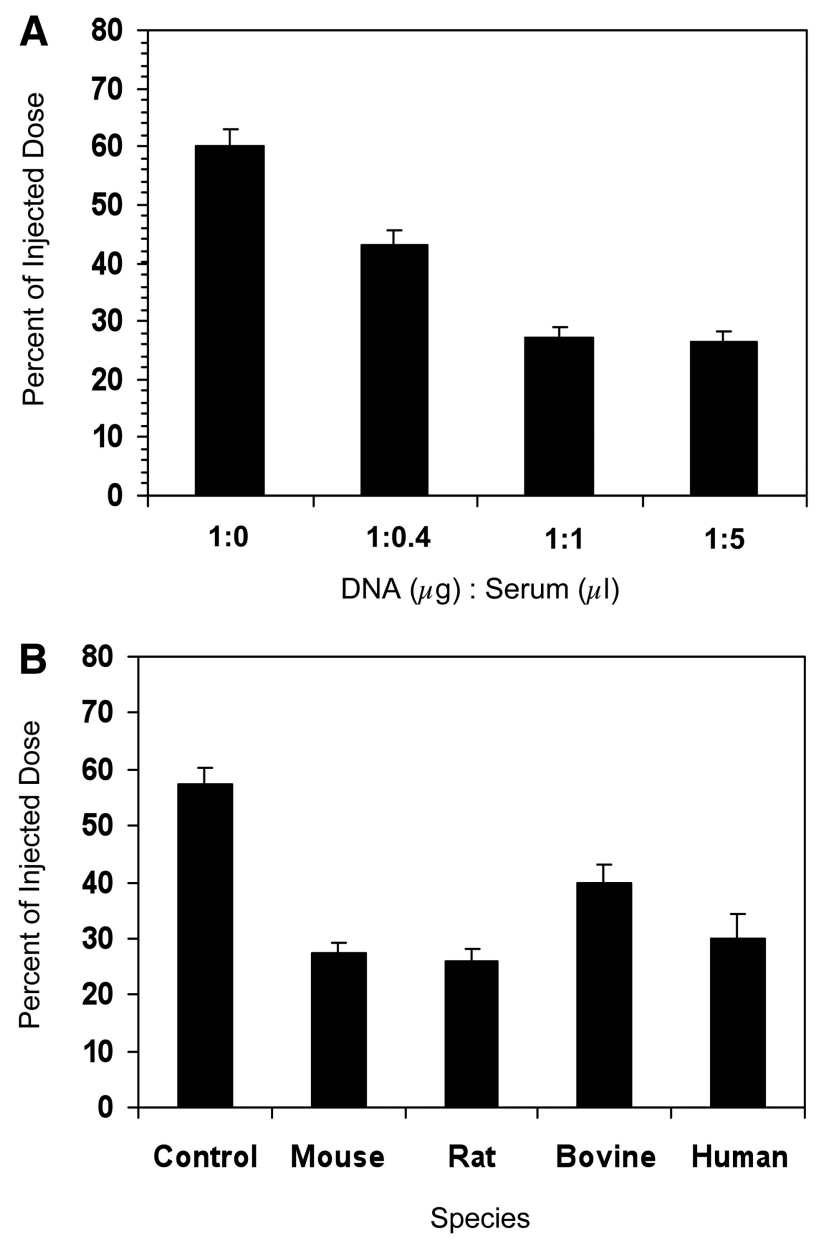

Fig. 2. a, inhibition of DNA uptake by the liver by use of various ratios of mouse serum to DNA $(p<0.05,0.01)$. b, inhibition of DNA uptake by the liver by use of various ratios of serum of other species.

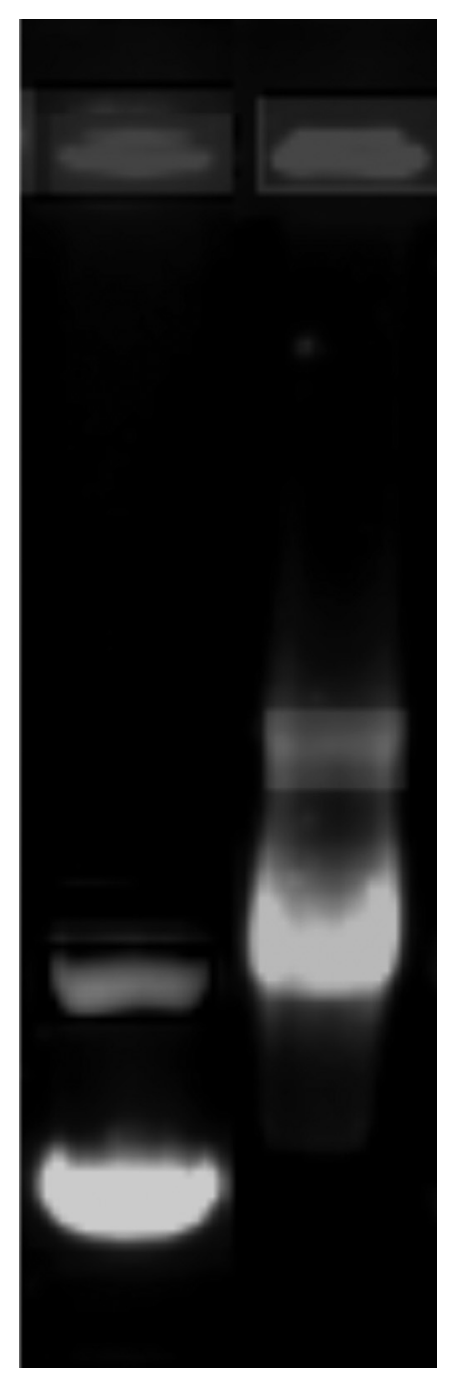

Fig. 3. Mobility shift assay of SDBPs and DNA complexes. The complexes form a distinct band in the right lane band characterized by decreased gel mobility compared with plasmid DNA alone in the left lane.

up by the liver and eliminated from blood. Presumably, if the binding condition is provided for SDBPs, their in vivo dysopsonin activity should be observed. We, therefore, designed in vivo studies to confirm the activity of serum diminishing liver uptake of DNA. The first study was to preincubate DNA with serum before the injection. Compared with a control, the DNA preincubated with serum (SDBPs/DNA) had a decrease in percentage taken up by the liver and an increase of percentage in the blood. The second study was to provide a retention time for SDBPs binding the injected DNA (see Materials and Methods), in which the DNA, after the injection, was allowed to circulate in blood for a few minutes but was not passed through the liver. By blocking the hepatic artery and portal vein, access to the liver was effectively impeded via the intravenous route, and serum proteins with dysopsonin properties in the mouse model were provided additional time to bind DNA before clamp release and liver uptake (Fig. 4). By doing so, an even greater percentage decrease in liver uptake was examined when the portal vein and hepatic artery were temporarily clamped in the in vivo model, followed by naked DNA administration (Fig. 4). One question may be raised that transiently blocking blood 


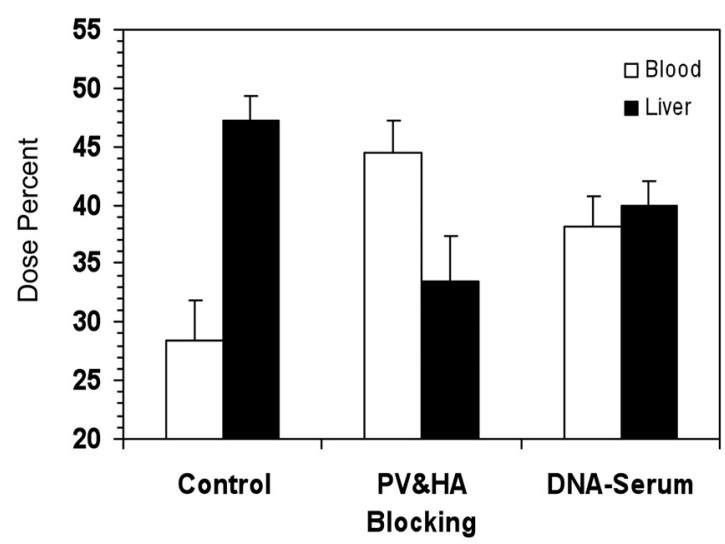

DNA Administration

Fig. 4. In vivo serum effect on DNA uptake. Compared with a control, DNA preincubated with serum had a decreased percent uptake in the liver in a mouse model. An even greater percentage decrease in liver uptake was visualized when the portal vein (PV) and hepatic artery (HA) were temporarily clamped, allowing serum proteins with dysopsonin properties additional time to bind DNA before clamp release and exposure to liver nonparenchymal cells.

through the liver may change liver function such that the uptake of DNA could be decreased. This possibility was ruled out by measuring the uptake of DNA that was intravenously injected after the performance of clamping and releasing the blood flow through the liver, which showed no difference in liver DNA uptake compared with that of intact mice (data not shown). We also transferred the SDBPs/DNA to the liver and lung to examine whether the gene transfer efficiency is affected by SDBPs. Data using hydrodynamic gene transfer in the mouse liver (Liu et al., 1999) and lung gene transfer with sequential injection (Song et al., 1998; Tan et al., 2001) revealed that serum proteins bound to DNA do not affect the biological activity of the plasmid DNA (Fig. 5). During sequential injection, cationic liposomes were injected into a mouse model followed by injection of DNA after a time interval. Cationic liposomes form aggregates after exposure to blood or serum because of electrostatic interactions, a consequence leading to trapped DNA after a second injection of a gene therapy product. Similar to our study in which blood flow was blocked through the liver, sequential injection minimized the flushing effect of blood in vivo and prolonged the exposure time of DNA to target cells for enhanced gene transfer in the lung (Song et al., 1998; Tan et al., 2001).

Identification of SDBPs. Next, we identified the SDBPs from mouse serum. We purified proteins with potential dysopsonin properties by use of dsDNA cellulose incubated

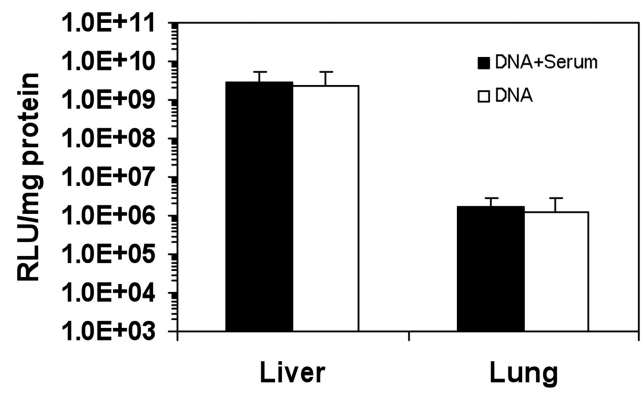

Fig. 5. Biological activity of plasmid DNA. Data using hydrodynamic gene transfer in the mouse liver and in situ transfection in mouse lung reveal that serum DNA-binding proteins do not affect the biological activity of plasmid DNA. RLU, relative light units. with mouse serum, followed by digestion with DNase I to release SDBPs. We initially had difficulty in completely eluting SDBPs from the dsDNA. After elution with 1 to $2 \mathrm{M} \mathrm{NaCl}$ per the GE Healthcare protocol, a substantial amount of SDBPs remained bound to the DNA-cellulose, detected via the Coomassie Plus Protein Reagent Kit (Pierce Chemical). A new method was developed to identify SDBPs, in which DNA (dsDNA-cellulose/mouse serum) was digested with DNase I to release SDBPs. Proteins were visualized by use of a SDSPAGE gel. In Fig. 6A, three dominant SDBP bands can be visualized in lane 2 (arrows a, b, and c) with sizes of approximately $18 \mathrm{kDa}, 20 \mathrm{kDa}$, and $240 \mathrm{kDa}$, which were not present in lanes 1 and 3 . Lanes 1 and 2 were from samples of cellulose/mouse serum (control) and dsDNA-cellulose/mouse serum, respectively. Lane 3 was from the sample of equal amounts of DNase used in lanes 1 and 2. Lane 4 was from mouse serum $(0.6 \mu \mathrm{l})$ alone. Similar bands of SDBPs were detected in human serum from two individuals in Fig. 6B (circled bands in lanes 5 and 6). The three major protein

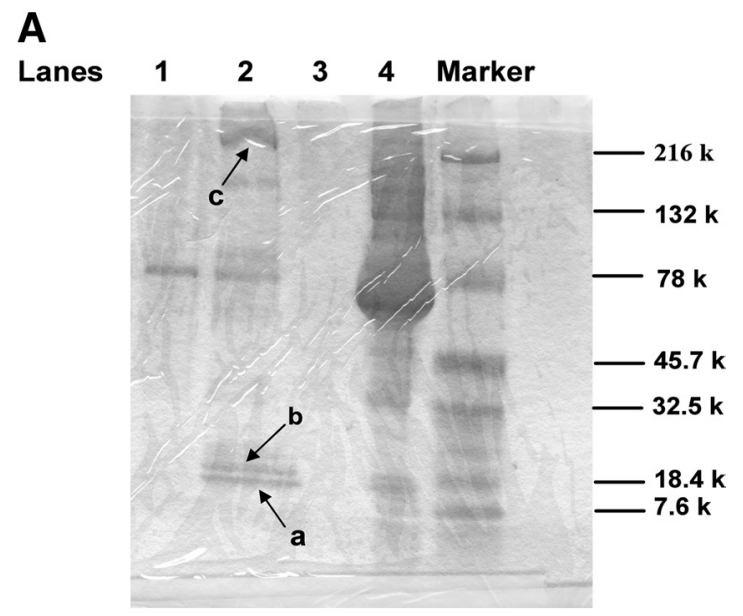

B

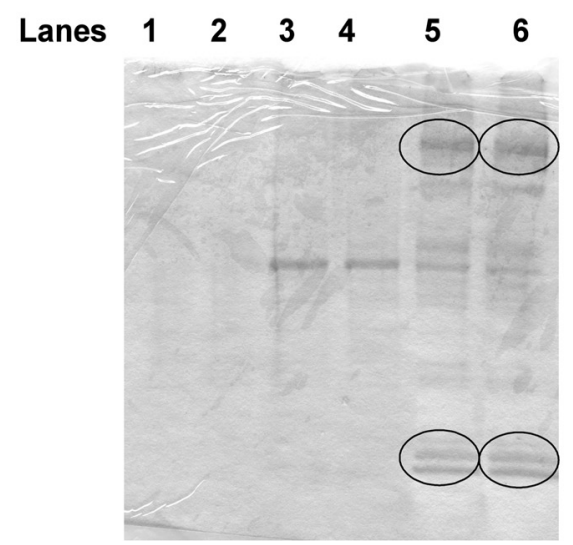

Fig. 6. A, SDS-PAGE analysis of serum DNA-binding proteins from mouse serum. Three dominant SDBPs can be visualized in lane 2 containing the dsDNA-cellulose/mouse serum with approximate sizes of 18 $\mathrm{kDa}(\mathrm{c}), 20 \mathrm{kDa}(\mathrm{b})$, and $240 \mathrm{kDa}$ (c). Lane 1 contains the cellulose/mouse serum control, whereas lane 3 is from the sample of equal amounts of DNase used in lanes 1 and 2. Lane 4 is from the mouse serum $(0.6 \mu \mathrm{l})$ alone. B, SDS-PAGE analysis of serum DNA-binding proteins from human serum. Lanes 1 and 2 contain DNase, whereas lanes 3 and 4 contain the cellulose/human serum control. Lanes 5 and 6 are from dsDNAcellulose/human serum. Similar bands of serum DNA-binding proteins identified with arrows $\mathrm{a}, \mathrm{b}$, and $\mathrm{c}$ in A were detected with human serum from two individuals. 
bands from Fig. 6A were identified with mass spectrophotometry analysis. Identified SDBPs included platelet factor 4, histone 4 , cytoplasmic $\beta$-actin, $\beta 1$-globin, albumin 1 , thrombospondin 1 precursor, hemoglobin $\alpha$-chains, and histonelike proteins.

\section{Discussion}

In general, intravenous injection of naked plasmid DNA results in no gene expression, even in the liver where the highest uptake of gene delivery occurs. It has been suggested that some SDBPs exist as scavengers to remove DNA from the serum. We hypothesized that SDBPs would bind to injected plasmid DNA, labeling plasmid DNA as foreign for uptake by liver nonparenchymal cells. Contrary to the original hypothesis, additional data indicate that some SDBPs may have important dysopsonin properties, acting to reduce liver uptake, and protective properties against blood degradation of plasmid DNA. Therefore, we proposed a unique nonviral gene delivery system by use of serum proteins with dysopsonin properties. Extending the in vivo circulation time of plasmid DNA for delivery is instrumental to gene therapy, because plasmid DNA must be protected from degradation when systemically administered. Use of innate serum proteins with dysopsonin properties may also enhance the potential for systemic gene delivery by reducing toxicity, in particular, immunogenicity, and through ease of amplification. Another advantage to using serum proteins with dysopsonin properties is that the biological activity of the plasmid DNA does not seem to be compromised, as evidenced through hydrodynamic gene transfer in the mouse liver and in situ transfection in the mouse lung.

Although nonviral liposomal vectors have been designed for systemic gene therapy with increased stability in the bloodstream, problems such as aggregation, inflammatory toxicity, and gene delivery inefficiency exist. Gene delivery methods should ideally protect against degradation by nucleases, transport the gene across plasma and nuclear membranes, and be devoid of harmful effects (Gao et al., 2007). In this study, we identified SDBPs with potential dysopsonin properties including platelet factor 4 , histone 4 , cytoplasmic $\beta$-actin, $\beta 1$-globin, albumin 1 , thrombospondin 1 precursor, hemoglobin $\alpha$-chains, and histone-like proteins. An optimal gene delivery protein candidate for conjugation to DNA must be isolated and further amplified through a bacterial vector. The SDBP with dysopsonin properties and DNA complexes may be modified further through measures such as polyethylene glycolation and conjugation with targeting ligands to enhance specificity and cellular uptake for DNA transfer, while minimiz- ing toxicity in vivo after identification of essential protein binding elements. These complexes ultimately may be developed into a novel DNA carrier system favorable for systemic gene delivery with prolonged circulation time as a result of decreased degradation.

\section{References}

Budker V, Zhang G, Danko I, Williams P, and Wolff J (1998) The efficient expression of intravascularly delivered DNA in rat muscle. Gene Ther 5:272-276.

Gao X, Kim K, and Liu D (2007) Nonviral gene delivery: what we know and what is next. AAPS J 9:E92-E104

Hisazumi J, Kobayashi N, Nishikawa M, and Takakura Y (2004) Significant role of liver sinusoidal endothelial cells in hepatic uptake and degradation of naked plasmid DNA after intravenous injection. Pharmacol Res 21:1223-1228.

Hofman CR, Dileo JP, Li Z, Li S, and Huang L (2001) Efficient in vivo gene transfer by PCR amplified fragment with reduced inflammatory activity. Gene Ther 8:71-74.

Kawabata K, Takakura Y, and Hashida M (1995) The fate of plasmid DNA after intravenous injection in mice: involvement of scavenger receptors in its hepatic uptake. Pharmacol Res 12:825-830.

Kobayashi N, Kuramoto T, Yamaoka K, Hashida M, and Takakura Y (2001) Hepatic uptake and gene expression mechanisms following intravenous administration of plasmid DNA by conventional and hydrodynamics-based procedures. J Pharmacol Exp Ther 297:853-860.

Lecocq M, Andrianaivo F, Warnier MT, Wattiaux-De Coninck S, Wattiaux R, and Jadot M (2003) Uptake by mouse liver and intracellular fate of plasmid DNA after a rapid tail vein injection of a small or a large volume. J. Gene Med 5:142-156.

Liu D, Liu F, and Song YK (1995) Recognition and clearance of liposomes containing phosphatidylserine are mediated by serum opsonin. Biochim Biophys Acta 1235: $140-146$.

Liu F and Huang L (2002) Noninvasive gene delivery to the liver by mechanical massage. Hepatology 35:1314-1319.

Liu F, Shollenberger LM, Conwell CC, Yuan X, and Huang L (2007) Mechanism of DNA clearance after intravenous injection. J Gene Med 9:613-619.

Liu F, Song Y, and Liu D (1999) Hydrodynamics-based transfection in animals by systemic administration of plasmid DNA. Gene Ther 6:1258-1266.

Moghimi SM and Patel HM (1989) Serum opsonins and phagocytosis of saturated and unsaturated phospholipid liposomes. Biochim Biophys Acta 984:384-387.

Nakamura M, Davila-Zavala P, Tokuda H, Takakura Y, and Hashida M (1998) Uptake and gene expression of naked plasmid DNA in cultured brain microvessel endothelial cells. Biochem Biophys Res Commun 245:235-239.

Sellins K, Fradkin L, Liggitt D, and Dow S (2005) Type I interferons potently suppress gene expression following gene delivery using liposome(-)DNA com plexes. Mol Ther 12:451-459.

Song YK, Liu F, and Liu D (1998) Enhanced gene expression in mouse lung by prolonging the retention time of intravenously injected plasmid DNA. Gene Ther 5:1531-1537.

Tan Y, Liu F, Li Z, Li S, and Huang L (2001) Sequential injection of cationic liposome and plasmid DNA effectively transfects the lung with minimal inflammatory toxicity. Mol Ther 3:673-682.

Yan X, Scherphof GL, and Kamps JA (2005) Liposome opsonization. J Liposome Res 15:109-139.

Yoshida M, Mahato R, Kawabata K, Takakura Y, and Hashida M (1996) Disposition characteristics of plasmid DNA in the single-pass rat liver perfusion system. Pharm Res 13:599-603.

Zhang G, Budker V, and Wolff JA (1999) High levels of foreign gene expression in hepatocytes after tail vein injections of naked plasmid DNA. Hum Gene Ther 10:1735-1737.

Zhang G, Gao X, Song YK, Vollmer R, Stolz DB, Gasiorowski JZ, Dean DA, and Liu $\mathrm{D}$ (2004) Hydroporation as the mechanism of hydrodynamic delivery. Gene Ther 11:675-682.

Zhang JS, Liu F, Conwell CC, Tan Y, and Huang L (2006) Mechanistic studies of sequential injection of cationic liposome and plasmid DNA. Mol Ther 13:429-437.

Address correspondence to: Dr. Feng Liu, Division of Molecular Pharmaceutics, Eshelman School of Pharmacy, University of North Carolina, 1318 Kerr Hall, CB 7360, Chapel Hill, NC 27599-7360. E-mail: fliu@email.unc.edu. 